\title{
Reanalysis of the most strange dibaryon within constituent quark models
}

\author{
Hongxia Huang,,$^{1, *}$ Xinmei Zhu, ${ }^{2, \dagger}$ and Jialun Ping $\oplus^{1, \ddagger}$ \\ ${ }^{1}$ Department of Physics and Jiangsu Key Laboratory for Numerical Simulation of Large Scale Complex Systems, \\ Nanjing Normal University, Nanjing 210023, People's Republic of China \\ ${ }^{2}$ Department of Physics, Yangzhou University, Yangzhou 225009, People's Republic of China
}

(Received 25 December 2019; accepted 25 February 2020; published 31 March 2020)

\begin{abstract}
The most strange dibaryon $\Omega \Omega$ with quantum numbers $S=-6, I=0$, and $J^{P}=0^{+}, 1^{-}, 2^{+}, 3^{-}$is reanalyzed in the framework of a quark delocalization color screening model (QDCSM) and chiral quark model (ChQM). The $\Omega \Omega$ dibaryon with $J^{P}=0^{+}$is bound, and the one with other quantum numbers $J^{P}=1^{-}, 2^{+}, 3^{-}$ are all unbound in our calculation. The low-energy scattering phase shifts, the scattering length, and the effective range of the $\Omega \Omega$ dibaryon with $J^{P}=0^{+}$also support the existence of such strange dibaryon. This dibaryon is showed to be a shallow bound state in QDCSM, while the binding energy becomes much larger in the ChQM by including the effect of the hidden color channels coupling. And the scalar nonet meson exchange in the ChQM also provides more attraction for the $\Omega \Omega$ system. Experimental search for such most strange dibaryon will provide much information for understanding the hadron-hadron interactions in different quark models.
\end{abstract}

DOI: 10.1103/PhysRevC.101.034004

\section{INTRODUCTION}

It is commonly believed that quantum chromodynamics (QCD) is the fundamental theory of the strong interaction. However, the low-energy physics of QCD, such as the structure of hadrons, hadron-hadron interactions, and the structure of multiquark systems, is hard to calculate directly from QCD. Various QCD-inspired quark models have been developed to obtain physical insights into multiquark systems, such as the MIT bag model [1], cloudy bag model [2], Friedberg-Lee nontopological soliton model [3], Skyrme topological soliton model [4], the constituent quark model [5,6], etc. Different models use quite different effective degrees of freedom, which might be indicative of the nature of low-energy QCD.

The constituent quark model has been quite successful in understanding hadron spectroscopy and hadron-hadron interactions even though one has not yet derived the constituent quark model directly from QCD. De Rujula, Georgi, and Glashow [5] first put forward a quark-gluon coupling model based on constituent quark and gluon effective degrees of freedom. Isgur and Karl obtained a good description of hadron spectroscopy based on this model [6]. However, extension of the model to baryon-baryon interactions does not reproduce

\footnotetext{
*hxhuang@njnu.edu.cn

${ }^{\dagger}$ zxm_yz@126.com

${ }^{\ddagger}$ Corresponding author: jlping@njnu.edu.cn
}

Published by the American Physical Society under the terms of the Creative Commons Attribution 4.0 International license. Further distribution of this work must maintain attribution to the author(s) and the published article's title, journal citation, and DOI. Funded by $S C O A P^{3}$. the nucleon-nucleon $(N N)$ intermediate and long-range interaction.

One modification is the addition of scalar meson exchange and Goldstone bosons exchange on the quark level [7-10], which provide the nucleon-nucleon intermediate and longrange interaction, respectively. A typical approach is the chiral quark model (ChQM) [11,12], in which the constituent quarks interact with each other through colorless Goldstone bosons exchange in addition to the colorful one-gluon exchange and confinement. To obtain the immediate-range attraction of the $N N$ interaction, the chiral partner $\sigma$ meson exchange has to be introduced. The $\sigma$ meson had been observed by the BES Collaboration as a $\pi \pi S$-wave resonance [13]. However, the results found by three groups independently show that the correlated two-pion exchange between two nucleons generates strong short-range repulsion and very moderate longrange attraction, which is quite different from the behavior of the $\sigma$ meson which used in the ChQM [14]. Therefore, one may wonder whether the $\sigma$ meson used in the ChQM is the correlated $\pi \pi$ resonance or an effective one.

An alternative approach to study baryon-baryon interaction is the quark delocalization color screening model (QDCSM), which was developed in 1990s with the aim of explaining the similarities between nuclear and molecular forces [15]. Two new ingredients were introduced: quark delocalization (to enlarge the model variational space to take into account the mutual distortion or the internal excitations of nucleons in the course of their interactions, the distortion of wave functions in the existence of other nucleons is also considered in the quarkmeson-coupling model [16]) and color screening (assuming the quark-quark interaction dependent on quark states aimed to take into account the QCD effect which has not yet been included in the two-body confinement and effective one gluon exchange). The model gives a good description of $N N$ and 
$Y N$ interactions and the properties of deuteron [17,18]. It is also employed to calculate the baryon-baryon scattering phase shifts and predict the dibaryon candidates $d^{*}$ and $N \Omega$ [19-24].

The difference between the ChQM and QDCSM is the intermediate-range attraction mechanism, which is the $\sigma$ meson exchange in ChQM and the quark delocalization and color screening in QDCSM. These two models have been applied to the study of $N N$ and $N \Omega$ systems $[18,19,23,24]$. The results show that the intermediate-range attraction mechanism in the QDCSM is equivalent to $\sigma$ meson exchange in the ChQM in these two systems. Moreover, the color screening is an effective description of the hidden color channels coupling [25]. It is interesting to check this consistency in other systems, such as the most strange dibaryon $\Omega \Omega$.

The dibaryon $\Omega \Omega$ with quantum numbers $S=-6, I=0$, and $J=0$ was predicted by Kopeliovich in the framework of the flavor SU(3) Skyrmion model [26]. Zhang et al. also suggested to search for this $\Omega \Omega$ state in heavy-ion collision experiments [27]. And this dibaryon was also showed to be a bound state in the QDCSM [28]. Very recently, $\Omega \Omega$ with $S=-6, I=0$, and $J=0$ was investigated by the HAL QCD Collaboration [29]. They studied this dibaryon on the basis of the $(2+1)$-flavor lattice QCD simulations with a nearly physical pion mass $m_{\pi} \simeq 146 \mathrm{MeV}$. The results showed that this $\Omega \Omega$ state had an overall attraction and was located near the unitary regime. They suggested that such a system can be best searched experimentally by the pair-momentum correlation in relativistic heavy-ion collisions. Then Morita et al. [30] calculated the correlation functions of this $\Omega \Omega$ state based on an expanding source model by using the interaction potentials from the lattice QCD calculations.

In this work, we reanalyze the most strange dibaryon $\Omega \Omega$ with quantum numbers $S=-6, I=0$, and $J^{P}=$ $0^{+}, 1^{-}, 2^{+}, 3^{-}$in both ChQM and QDCSM. The binding energy, as well as the low-energy scattering phase shifts, the scattering length, and the effective range, which are useful for the experimental search of this strange dibaryon, are investigated. By comparing the results within these two quark models, one can check the model dependence of this dibaryon. On the other hand, one can also inspect the consistency of the intermediate-range attraction mechanism of these two models in the dibaryon $\Omega \Omega$ system.

The structure of this paper is as follows. A brief introduction of two quark models is given in Sec. II. Section III is devoted to the numerical results and discussions. The summary is shown in the last section.

\section{TWO QUARK MODELS}

\section{A. Chiral quark model}

In this work, the Salamanca model was chosen as the representative of the ChQM, because the work of the Salamanca group covers the hadron spectra and the nucleon-nucleon interaction and has been extended to the study of multiquark states. The model details can be found in Ref. [12]. Here only the Hamiltonian is given:

$$
\begin{aligned}
H= & \sum_{i=1}^{6}\left(m_{i}+\frac{p_{i}^{2}}{2 m_{i}}\right)-T_{\mathrm{c} . \mathrm{m} .}+\sum_{j>i=1}^{6}\left(V_{i j}^{C}+V_{i j}^{G}+V_{i j}^{\chi}+V_{i j}^{\sigma}\right), \\
V_{i j}^{C}= & -a_{c} \lambda_{i}^{c} \cdot \lambda_{j}^{c}\left(r_{i j}^{2}+v_{0}\right), \\
V_{i j}^{G}= & \frac{1}{4} \alpha_{s} \lambda_{i}^{c} \cdot \lambda_{j}^{c}\left[\frac{1}{r_{i j}}-\frac{\pi}{2} \delta\left(\boldsymbol{r}_{i j}\right)\left(\frac{1}{m_{i}^{2}}+\frac{1}{m_{j}^{2}}+\frac{4 \sigma_{i} \cdot \boldsymbol{\sigma}_{j}}{3 m_{i} m_{j}}\right)-\frac{3}{4 m_{i} m_{j} r_{i j}^{3}} S_{i j}\right], \\
V_{i j}^{\chi}= & V_{\pi}\left(\boldsymbol{r}_{i j}\right) \sum_{a=1}^{3} \lambda_{i}^{a} \cdot \lambda_{j}^{a}+V_{K}\left(\boldsymbol{r}_{i j}\right) \sum_{a=4}^{7} \lambda_{i}^{a} \cdot \lambda_{j}^{a}+V_{\eta}\left(\boldsymbol{r}_{i j}\right)\left[\left(\lambda_{i}^{8} \cdot \lambda_{j}^{8}\right) \cos \theta_{P}-\left(\lambda_{i}^{0} \cdot \lambda_{j}^{0}\right) \sin \theta_{P}\right] \\
V_{\chi}\left(\boldsymbol{r}_{i j}\right)= & \frac{g_{\mathrm{ch}}^{2}}{4 \pi} \frac{m_{\chi}^{2}}{12 m_{i} m_{j}} \frac{\Lambda_{\chi}^{2}}{\Lambda_{\chi}^{2}-m_{\chi}^{2}} m_{\chi}\left\{\left(\boldsymbol{\sigma}_{i} \cdot \boldsymbol{\sigma}_{j}\right)\left[Y\left(m_{\chi} r_{i j}\right)-\frac{\Lambda_{\chi}^{3}}{m_{\chi}^{3}} Y\left(\Lambda_{\chi} r_{i j}\right)\right]\right. \\
& \left.+\left[H\left(m_{\chi} r_{i j}\right)-\frac{\Lambda_{\chi}^{3}}{m_{\chi}^{3}} H\left(\Lambda_{\chi} r_{i j}\right)\right] S_{i j}\right\}, \quad \chi=\pi, K, \eta, \\
V_{i j}^{\sigma}= & -\frac{g_{\mathrm{ch}}^{2}}{4 \pi} \frac{\Lambda_{\sigma}^{2}}{\Lambda_{\sigma}^{2}-m_{\sigma}^{2}} m_{\sigma}\left[Y\left(m_{\sigma} r_{i j}\right)-\frac{\Lambda_{\sigma}}{m_{\sigma}} Y\left(\Lambda_{\sigma} r_{i j}\right)\right], \\
S_{i j}= & \left\{3 \frac{\left(\boldsymbol{\sigma}_{i} \cdot \boldsymbol{r}_{i j}\right)\left(\boldsymbol{\sigma}_{j} \cdot \boldsymbol{r}_{i j}\right)}{r_{i j}^{2}}-\boldsymbol{\sigma}_{i} \cdot \boldsymbol{\sigma}_{j}\right\}, \\
H(x)= & \left(1+3 / x+3 / x^{2}\right) Y(x), \quad Y(x)=e^{-x} / x,
\end{aligned}
$$


where $\alpha_{s}$ is the quark-gluon coupling constant. In order to cover the wide energy scale from light to strange quark, one introduces an effective scale-dependent quark-gluon coupling constant $\alpha_{s}(\mu)$ [31],

$$
\alpha_{s}(\mu)=\frac{\alpha_{0}}{\ln \left(\frac{\mu^{2}+\mu_{0}^{2}}{\Lambda_{0}^{2}}\right)},
$$

where $\mu$ is the reduced mass of the interacting quark pair. The coupling constant $g_{\text {ch }}$ for chiral field is determined from the $N N \pi$ coupling constant through

$$
\frac{g_{\mathrm{ch}}^{2}}{4 \pi}=\left(\frac{3}{5}\right)^{2} \frac{g_{\pi N N}^{2}}{4 \pi} \frac{m_{u, d}^{2}}{m_{N}^{2}} .
$$

The other symbols in the above expressions have their usual meanings.

For the most strange dibaryons, two versions of ChQM $[32,33]$ are used here. One is the SU(2) ChQM, in which $\sigma$ meson is restricted to exchange between $u$ and/or $d$ quark pair only; another is the SU(3) ChQM, where full SU(3) scalar nonet meson exchange was used. These scalar potentials have the same functional form as the one of SU(2) ChQM but a different SU(3) operator dependence [32], that is,

$$
\begin{aligned}
V_{i j}^{\sigma_{a}}= & V_{a_{0}}\left(\boldsymbol{r}_{i j}\right) \sum_{a=1}^{3} \lambda_{i}^{a} \cdot \lambda_{j}^{a}+V_{\kappa}\left(\boldsymbol{r}_{i j}\right) \sum_{a=4}^{7} \lambda_{i}^{a} \cdot \lambda_{j}^{a} \\
& +V_{f_{0}}\left(\boldsymbol{r}_{i j}\right) \lambda_{i}^{8} \cdot \lambda_{j}^{8}+V_{\sigma}\left(\boldsymbol{r}_{i j}\right) \lambda_{i}^{0} \cdot \lambda_{j}^{0} \\
V_{k}\left(\boldsymbol{r}_{i j}\right)= & -\frac{g_{\mathrm{ch}}^{2}}{4 \pi} \frac{\Lambda_{k}^{2} m_{k}}{\Lambda_{k}^{2}-m_{k}^{2}}\left[Y\left(m_{k} r_{i j}\right)-\frac{\Lambda_{k}}{m_{k}} Y\left(\Lambda_{k} r_{i j}\right)\right],
\end{aligned}
$$

with $k=a_{0}, \kappa, f_{0}$, or $\sigma$. Here the mixing between flavor singlet and octet is not considered, i.e., the exact $\mathrm{SU}(3)$ flavor symmetry is used. In this way the $\sigma$ meson is different from the one in $\mathrm{SU}(2)$ symmetry. After all, the $\sigma$ meson in the meson exchange is an effective one.

\section{B. Quark delocalization color screening model}

The Hamiltonian of QDCSM is almost the same as that of ChQM but with two modifications [15,17]: First, there is no $\sigma$ meson exchange in QDCSM, and, second, the screened color confinement is used between quark pairs resident in different baryon orbits. That is,

$$
V_{i j}^{C}=\left\{\begin{array}{ll}
-a_{c} \lambda_{i}^{c} \cdot \lambda_{j}^{c}\left(r_{i j}^{2}+v_{0}\right) & \text { if } i, j \text { in the same } \\
-a_{c} \lambda_{i}^{c} \cdot \lambda_{j}^{c}\left(\frac{1-e^{-\mu_{i j} r_{i j}^{2}}}{\mu_{i j}}+v_{0}\right) & \text { othoryon orbit }
\end{array},\right.
$$

where the color screening constant $\mu_{i j}$ is determined by fitting the deuteron properties, $N N$ scattering phase shifts, and $N \Lambda$ and $N \Sigma$ scattering cross sections, $\mu_{u u}=0.45, \mu_{u s}=0.19$, and $\mu_{s s}=0.08$, which satisfy the relation $\mu_{u s}^{2}=\mu_{u u} \mu_{s s}$. Because of the quark delocalization, meson-exchange effects are partly considered, the hard cutoff of meson exchange is used to avoid the double counting in the previous work [34,35].
TABLE I. The parameters of two models: $m_{\pi}=0.7 \mathrm{fm}^{-1}, m_{K}=$ $2.51 \mathrm{fm}^{-1}, m_{\eta}=2.77 \mathrm{fm}^{-1}, m_{\sigma}=3.42 \mathrm{fm}^{-1}, m_{a_{0}}=m_{\kappa}=m_{f_{0}}=$ $4.97 \mathrm{fm}^{-1}, \Lambda_{\pi}=4.2 \mathrm{fm}^{-1}, \Lambda_{K}=5.2 \mathrm{fm}^{-1}, \Lambda_{\eta}=5.2 \mathrm{fm}^{-1}, \Lambda_{\sigma}=$ $4.2 \mathrm{fm}^{-1}, \quad \Lambda_{a_{0}}=\Lambda_{\kappa}=\Lambda_{f_{0}}=5.2 \mathrm{fm}^{-1}, \quad g_{\mathrm{ch}}^{2} /(4 \pi)=0.54, \quad \theta_{p}=$ $-15^{0}$.

\begin{tabular}{lccc}
\hline \hline & QDCSM & SU(2) ChQM & SU(3) ChQM \\
\hline$b(\mathrm{fm})$ & 0.518 & 0.518 & 0.518 \\
$m_{u}(\mathrm{MeV})$ & 313 & 313 & 313 \\
$m_{d}(\mathrm{MeV})$ & 313 & 313 & 313 \\
$m_{s}(\mathrm{MeV})$ & 573 & 536 & 573 \\
$a_{c}(\mathrm{MeV})$ & 58.03 & 48.59 & 48.59 \\
$\mu_{u u}\left(\mathrm{fm}^{-2}\right)$ & 0.45 & - & - \\
$\mu_{u s}\left(\mathrm{fm}^{-2}\right)$ & 0.19 & - & - \\
$\mu_{s s}\left(\mathrm{fm}^{-2}\right)$ & 0.08 & - & - \\
$v_{0}\left(\mathrm{MeV}^{2}\right)$ & -1.2883 & -1.2145 & -0.961 \\
$\alpha_{0}$ & 0.510 & 0.510 & 0.583 \\
$\Lambda_{0}\left(\mathrm{fm}^{-1}\right)$ & 1.525 & 1.525 & 1.616 \\
$\mu_{0}(\mathrm{MeV})$ & 445.808 & 445.808 & 422.430 \\
\hline \hline
\end{tabular}

In the present work, the usual soft cutoff is employed. The variational approach can take care of the double counting problem.

The single-particle orbital wave functions in the ordinary quark cluster model are the left and right centered single Gaussian functions:

$$
\begin{aligned}
\phi_{\alpha}\left(S_{i}\right) & =\left(\frac{1}{\pi b^{2}}\right)^{\frac{3}{4}} e^{-\frac{\left(r-S_{i} / 2\right)^{2}}{2 b^{2}}}, \\
\phi_{\beta}\left(-S_{i}\right) & =\left(\frac{1}{\pi b^{2}}\right)^{\frac{3}{4}} e^{-\frac{\left(r+S_{i} / 2\right)^{2}}{2 b^{2}}} .
\end{aligned}
$$

The quark delocalization in QDCSM is realized by writing the single-particle orbital wave function as a linear combination of the left and right Gaussians:

$$
\begin{aligned}
\psi_{\alpha}\left(\boldsymbol{S}_{i}, \epsilon\right) & =\left[\phi_{\alpha}\left(\boldsymbol{S}_{i}\right)+\epsilon \phi_{\alpha}\left(-\boldsymbol{S}_{i}\right)\right] / N(\epsilon), \\
\psi_{\beta}\left(-\boldsymbol{S}_{i}, \epsilon\right) & =\left[\phi_{\beta}\left(-\boldsymbol{S}_{i}\right)+\epsilon \phi_{\beta}\left(\boldsymbol{S}_{i}\right)\right] / N(\epsilon), \\
N(\epsilon) & =\sqrt{1+\epsilon^{2}+2 \epsilon e^{-S_{i}^{2} / 4 b^{2}}},
\end{aligned}
$$

where $\epsilon\left(\boldsymbol{S}_{i}\right)$ is the delocalization parameter determined by the dynamics of the quark system rather than adjusted parameters. In this way, the system can choose its most favorable configuration through its own dynamics in a larger Hilbert space.

The parameters of these models are from our previous work on the $N \Omega$ system [24], and all parameters are listed in Table I. The calculated baryon masses in comparison with experimental values are shown in Table II. Here one can see that the spin

TABLE II. The masses of the ground-state baryons (in MeV).

\begin{tabular}{lcccccccc}
\hline \hline & $N$ & $\Delta$ & $\Lambda$ & $\Sigma$ & $\Sigma^{*}$ & $\Xi$ & $\Xi^{*}$ & $\Omega$ \\
\hline QDCSM & 939 & 1232 & 1124 & 1238 & 1360 & 1374 & 1496 & 1642 \\
SU(2) ChQM & 939 & 1232 & 1137 & 1245 & 1376 & 1375 & 1506 & 1620 \\
SU(3) ChQM & 939 & 1232 & 1123 & 1267 & 1344 & 1398 & 1475 & 1625 \\
Expt. & 939 & 1232 & 1116 & 1193 & 1385 & 1318 & 1533 & 1672 \\
\hline \hline
\end{tabular}




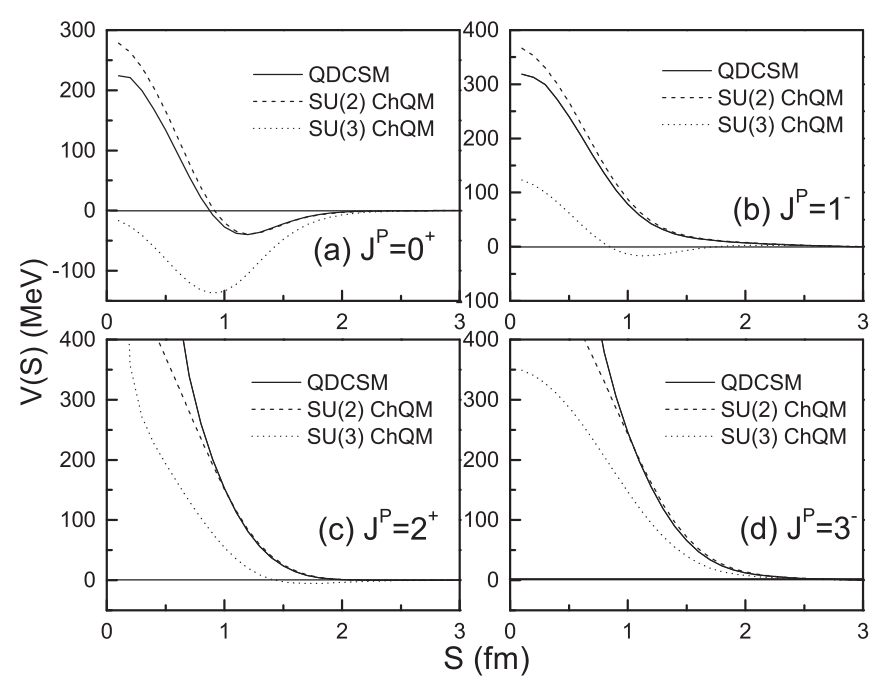

FIG. 1. The effective potentials of the $\Omega \Omega$ system in different quark models.

splittings of $\Sigma$ and $\Xi$ is smaller than that of the experimental data. This is due to the smaller value of the quark-gluon coupling constant $\alpha_{s}$ used here. The spin splittings mainly come from the color-magnetic interaction in the one-gluonexchange interaction [see Eq. (3)]. As mentioned above, we use an effective scale-dependent quark-gluon coupling constant $\alpha_{s}$ [see Eq. (9)] here to cover the wide energy scale from light to heavy quark. A better global fit of the hadron spectrum can be achieved in this way. If we adjust the parameter to give the right spin splittings of $\Sigma$ and $\Xi$, then the results for the $\Omega \Omega$ system are consistent with the present ones.

\section{RESULTS AND DISCUSSIONS}

\section{A. Effective potentials}

In this work, we investigate the most strange dibaryon $\Omega \Omega$ with quantum numbers $S=-6, I=0$, and $J^{P}=$ $0^{+}, 1^{-}, 2^{+}, 3^{-}$in QDCSM, SU(2) ChQM, and SU(3) ChQM. The partial wave of $J^{P}=0^{+}, 2^{+}$is $S$ wave, and the one of $J^{P}=1^{-}, 3^{-}$is $P$ wave. We calculate the effective potentials of the $\Omega \Omega$ system, because an attractive potential is necessary for forming bound state or resonance. The effective potential between two clusters is defined as $V(S)=E(S)-$ $E(\infty)$, where $E(S)$ is the diagonal matrix element of the Hamiltonian of the system in the generating coordinate. The effective potentials of $J^{P}=0^{+}, 1^{-}, 2^{+}, 3^{-}$in three quark models are shown in Figs. 1(a)-1(d), respectively.

From Fig. 1(a), one can see that the potentials are attractive for the $J^{P}=0^{+} \Omega \Omega$ state in all quark models. It is obvious that the attraction in $\mathrm{SU}(3) \mathrm{ChQM}$ is the largest one, followed by the attractions in SU(2) ChQM and QDCSM. For the $\Omega \Omega$ state with $J^{P}=1^{-}$, the potential is repulsive in SU(2) ChQM and QDCSM, but in SU(3) ChQM, it is a little bit attractive. The case is similar for the $\Omega \Omega$ state with $J^{P}=2^{+}$. For the $\Omega \Omega$ state with $J^{P}=3^{-}$, the potentials are all repulsive in three quark models. Therefore, from the behavior of the effective potentials of the $\Omega \Omega$ state, it is possible for the $J^{P}=0^{+} \Omega \Omega$
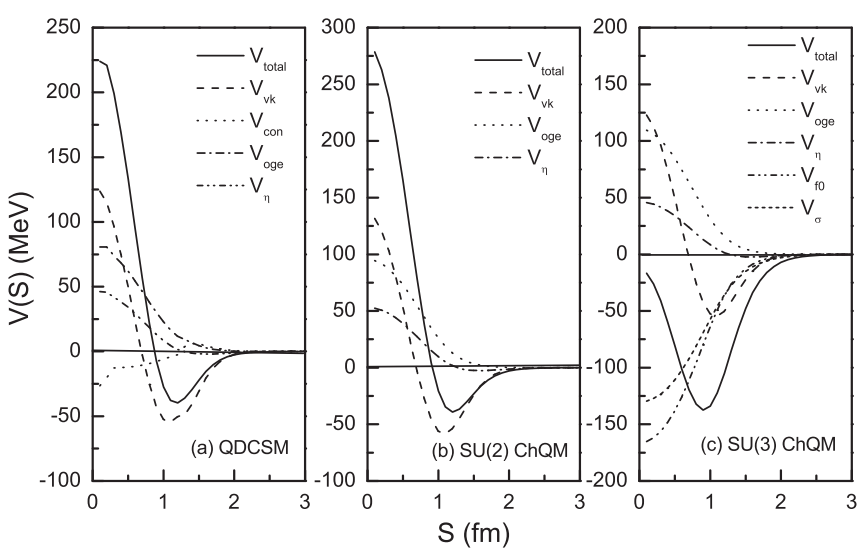

FIG. 2. The contributions to the effective potential from various terms of interactions for the $J^{P}=0^{+} \Omega \Omega$ state.

dibaryon to form bound state, while for the $\Omega \Omega$ dibaryon with other quantum numbers, it is nearly impossible to form any bound state because of the repulsive interaction between two $\Omega$ s.

In order to investigate the source of the attractions for the $J^{P}=0^{+} \Omega \Omega$ state, we calculate the contribution of each interaction term to the total potential of the system. The potentials of various terms: the kinetic energy $\left(V_{v k}\right)$, confinement $\left(V_{\text {con }}\right)$, one-gluon exchange $\left(V_{\text {oge }}\right)$, one-boson exchange $\left(V_{\pi}\right.$ and $V_{K}$ do not contribute to the effective potential because they do not exchange between two $s$ quarks, so only $V_{\eta}$ contributes), and scalar nonet meson exchange $\left(V_{\sigma}, V_{a_{0}}, V_{\kappa}\right.$, and $\left.V_{f_{0}}\right)$ are shown in Fig. 2.

For the QDCSM, quark delocalization and color screening work together to provide short-range repulsion and intermediate-range attraction. We illustrate this mechanism by showing contributions of all interaction terms to the effective potential in Fig. 2(a), from which one can see that the attraction of the $\Omega \Omega$ system mainly comes from the kinetic energy term. The confinement interaction provides a little attraction, while other terms provide repulsive potentials, which reduce the total attraction of the $\Omega \Omega$ potential.

For the SU(2) ChQM, the quadratic confinement does not contribute to the potential between two $\Omega$ 's because of the properties of two color singlets. Since the $\sigma$ meson is restricted to exchange between the $u$ and $d$ quarks only in SU(2) $\mathrm{ChQM}$, there is no $\sigma$ meson-exchange interaction between two $\Omega$ s. Therefore, only kinetic energy, one-gluon-exchange, and one- $\eta$-exchange contribute to the effective potentials. It is shown in Fig. 2(b) that the kinetic energy term provides the major attraction, while other two terms provide repulsive potentials, which decrease the total attractions.

For the SU(3) ChQM, scalar nonet meson exchange is included. Although $a_{0}$ and $\kappa$ mesons do not contribute because they do not exchange between $s$ quarks, both $f_{0}$ meson exchange and $\sigma$ meson exchange introduce large attractions, which lead to the strong attraction between two $\Omega$ s.

\section{B. Binding energy calculation}

In order to see whether there is any bound state, we carry out a dynamic calculation. The resonating group method 
TABLE III. The binding energies (in MeV) of the $J^{P}=0^{+} \Omega \Omega$ system in different models.

\begin{tabular}{lcc}
\hline \hline & \multicolumn{1}{c}{$B_{\text {sc }}$} & \multicolumn{1}{c}{$B_{\mathrm{cc}}$} \\
\hline QDCSM & -2.0 & $\ldots$ \\
SU(2) ChQM & -0.7 & -46.8 \\
SU(3) ChQM & -79.9 & -103.3 \\
\hline
\end{tabular}

(RGM), described in more detail in Ref. [36], is used here. Expanding the relative motion wave function between two clusters in the RGM by a set of Gaussians, the integrodifferential equation of RGM can be reduced to algebraic equation, the generalized eigenequation. The energy of the system can be obtained by solving the eigenequation. In the calculation, the baryon-baryon separation $\left(\left|\mathbf{s}_{n}\right|\right)$ is taken to be less than 6 fm (to keep the matrix dimension manageably small).

In our calculation, the $\Omega \Omega$ system with $J^{P}=1^{-}, 2^{+}, 3^{-}$ are unbound in all quark models, which agree with the repulsive nature of the interaction of these states, while the $J^{P}=0^{+} \Omega \Omega$ state is bound in all quark models, due to the strong attractions in this system. Here we discuss the $J^{P}=0^{+}$ state in detail. The binding energies of the $J^{P}=0^{+} \Omega \Omega$ state in various quark models are listed in Table III, where $B_{\mathrm{sc}}$ denotes the binding energy of the single channel $\Omega \Omega$ and $B_{\mathrm{cc}}$ refers to the binding energy with the hidden color channels coupling.

The single-channel calculation shows that the binding energy in QDCSM and SU(2) ChQM is very small, which indicates that the $J^{P}=0^{+} \Omega \Omega$ is a shallow bound state. In contrast, the binding energy in the $\mathrm{SU}(3) \mathrm{ChQM}$ is much larger due to the stronger attraction between two $\Omega$ s, which suggests that the $\Omega \Omega$ is a deep bound state. By coupling the hidden color channels, a much deeper binding energy is obtained in both the SU(2) ChQM and SU(3) ChQM, which reaches $-46.8 \mathrm{MeV}$ and $-103.3 \mathrm{MeV}$, respectively. It indicates that the effect of the hidden color channels coupling is important for the $\Omega \Omega$ system in the ChQM. In QDCSM, since it contains the hidden color channels coupling effect already through the color screening $[19,25]$, including the color-singlet channels is enough. Therefore, one find that the $J^{P}=0^{+} \Omega \Omega$ appears as a shallow bound state in QDCSM, and this conclusion is consistent with that of the HAL QCD Collaboration [29], in which they showed that the $J^{P}=0^{+} \Omega \Omega$ state had an overall attraction and was located near the unitary regime. However, the $J^{P}=0^{+} \Omega \Omega$ state becomes a deeper bound state in the SU(2) ChQM and even a much deeper bound state in the SU(3) ChQM.

\section{The low-energy scattering phase shifts}

For the purpose of providing more information for the experimental search of such most strange dibaryon, we calculate the low-energy scattering phase shifts, the scattering length, and the effective range of the $\Omega \Omega$ dibaryon with $J^{P}=0^{+}$. In experiment, each $\Omega$ can be identified through a successive weak decay $\Omega^{-} \rightarrow \Lambda+K^{-} \rightarrow p+\pi^{-}+K^{-}$. A large scattering length (not the existence of a bound state) is the important element for the correlation $C(Q)$ to have

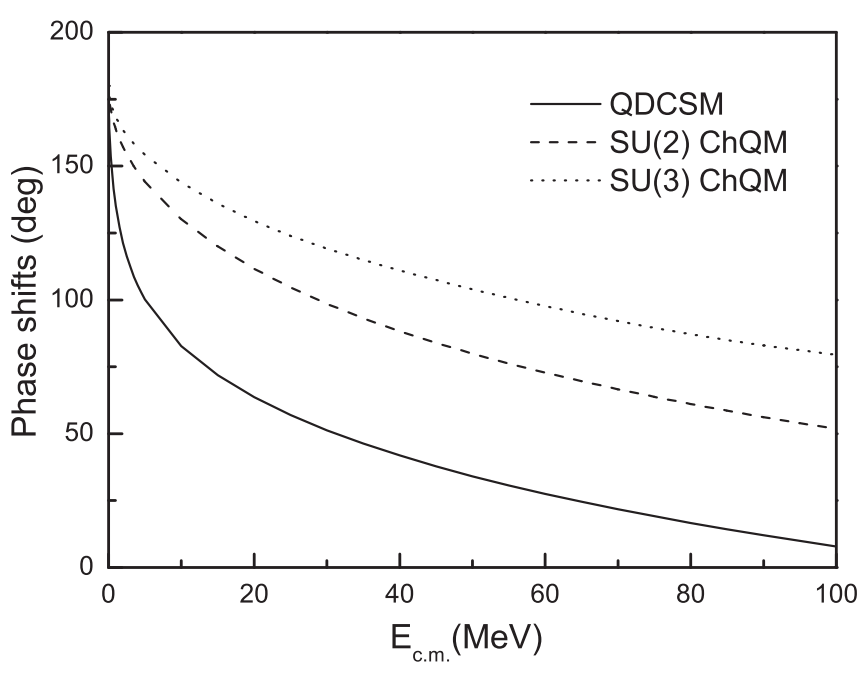

FIG. 3. The phase shifts of the $J^{P}=0^{+} \Omega \Omega$ state.

characteristic enhancement at small relative momentum $Q$ [37]. Here the well-developed Kohn-Hulthen-Kato variational method is used to calculate the low-energy scattering phase shifts. The details can be found in Ref. [36]. Figure 3 illustrates the scattering phase shifts of the $J^{P}=0^{+} \Omega \Omega$ state. It is obvious that in all quark models, the scattering phase shifts go to $180^{\circ}$ at $E_{\text {c.m. }} \sim 0$ and rapidly decreases as $E_{\text {c.m. }}$. increases, which implies the existence of a bound state. The results are consistent with the the bound-state calculation shown above. Besides, the behavior of the low-energy scattering phase shifts is also in agreement with that of the lattice QCD calculation [29].

Then, the scattering length $a_{0}$ and the effective range $r_{0}$ of the $\Omega \Omega$ state can be extracted from the low-energy scattering phase shifts by the following formula:

$$
k \cot \delta=-\frac{1}{a_{0}}+\frac{1}{2} r_{0} k^{2}+\mathcal{O}\left(k^{4}\right),
$$

where $\delta$ is the low-energy scattering phase shifts, $k$ is the momentum of the relative motion with $k=\sqrt{2 \mu E_{\mathrm{c} . \mathrm{m} .}}, \mu$ is the reduced mass of two baryons, and $E_{\mathrm{c} . \mathrm{m}}$. is the incident energy. The binding energy $B^{\prime}$ can be calculated according to the relation:

$$
B^{\prime}=\frac{\hbar^{2} \alpha^{2}}{2 \mu},
$$

where $\alpha$ is the wave number, which can be obtained from the relation [38]:

$$
r_{0}=\frac{2}{\alpha}\left(1-\frac{1}{\alpha a_{0}}\right) .
$$

The results are listed in Table IV.

From Table IV, one can see that in all quark models, the scattering length are all positive, which implies that the $J^{P}=0^{+} \Omega \Omega$ dibaryon is a bound state here. The binding energies obtained by Eq. (16) are broadly consistent with that in Table III, which is obtained by the dynamic calculation. Here again, the scattering length $a_{0}$ and effective range $r_{0}$ of the $J^{P}=0^{+} \Omega \Omega$ dibaryon in the QDCSM are in agreement 
TABLE IV. The scattering length $a_{0}$, effective range $r_{0}$, and binding energy $B^{\prime}$ of the $J^{P}=0^{+} \Omega \Omega$ dibaryon.

\begin{tabular}{llll}
\hline \hline & $a_{0}(\mathrm{fm})$ & $r_{0}(\mathrm{fm})$ & $B^{\prime}(\mathrm{MeV})$ \\
\hline QDCSM & 4.1296 & 1.3977 & -0.31 \\
SU(2) ChQM & 1.4050 & 0.6813 & -35.6 \\
SU(3) ChQM & 0.87275 & 0.42850 & -92.3 \\
\hline \hline
\end{tabular}

with the results of the lattice QCD calculation [29], in which $a_{0}=4.6(6)\left({ }_{-0.5}^{+1.2}\right) \mathrm{fm}$ and $r_{0}=1.27(3)\left({ }_{-0.03}^{+0.06}\right) \mathrm{fm}$.

\section{SUMMARY}

In summary, we reanalyze the most strange dibaryon $\Omega \Omega$ with quantum numbers $S=-6, \quad I=0$, and $J^{P}=$ $0^{+}, 1^{-}, 2^{+}, 3^{-}$in QDCSM, SU(2) ChQM, and SU(3) ChQM. Our results show the following: (1) The $\Omega \Omega$ with quantum numbers $J^{P}=1^{-}, 2^{+}, 3^{-}$are all unbound, due to the repulsive interaction between two $\Omega \mathrm{s}$ in these systems. (2) For the $J^{P}=0^{+} \Omega \Omega$, the attraction between two $\Omega \mathrm{s}$ is strong enough to form a bound state in all quark models. (3) The low-energy scattering phase shifts, the scattering length, and the effective range of the $\Omega \Omega$ system with $J^{P}=0^{+}$also support that this most strange dibaryon is a bound state. (4) All the results in the QDCSM are consistent with that obtained by the HAL QCD method, which suggest that the $J^{P}=0^{+} \Omega \Omega$ dibaryon is a shallow bound state. In contract, the binding energy is much deeper in both the SU(2) ChQM $\mathrm{SU}$ (3) ChQM, where the hidden color channels coupling is employed.

The quark model study of the hadron interaction has experienced a long history. The mechanism of the intermediaterange attraction of the baryon-baryon interaction is one of the most important issues in the study. In the $N N$ case we have shown that the phenomenological $\sigma$-meson exchange in ChQM is equivalent to the quark delocalization and color screening in QDCSM [18] and the color screening effect in QDCSM is an effective description of hidden color channels coupling [25]. For the strange $N \Omega$ system, the QDCSM predicts a bound $N \Omega$ dibaryon with quantum numbers $S=-3$, $I=\frac{1}{2}, J^{P}=2^{+}$, while the ChQM cannot obtain the bound state if the $\sigma$ meson is not universally exchanged between any quark pair. However, the bound state was finally obtained by considering the the hidden color channels coupling. Although similar results are obtained in both models, the mechanism is different. In QDCSM, quark delocalization and color screening work together to provide short-range repulsion and intermediate-range attraction, and the coupling of the color singlet channels is enough to form a bound state $N \Omega$, while in SU(3) ChQM, although the universal $\sigma$-meson exchange introduces large attraction, it is canceled by the repulsive potentials of $\kappa$ and $f_{0}$ exchange, and the bound $N \Omega$ state is obtained by coupling both color singlet and hidden color channels. Extending to the most strange $\Omega \Omega$ dibaryon, a shallow bound state is obtained in QDCSM. But this $J^{P}=0^{+} \Omega \Omega$ becomes a much deeper bound state in both SU(2) ChQM and SU(3) ChQM by coupling the hidden color channels. Besides, in SU(3) ChQM, the $f_{0}$ meson exchange and $\sigma$ meson exchange introduce large attractions which also increase the total attraction of the $\Omega \Omega$ potential.

To validate the intermediate attraction mechanism, more experimental data are needed. Experimental search for dibaryons may provide more information for this issue. The STAR experiment has made considerable headway in searching for the $N \Omega$ bound state [39]. If experiment confirms the existence of the $N \Omega$ dibaryon state, then it will be a signal showing that quark delocalization and color screening (an effective description of hidden color channels coupling) are effective ways to describe the intermediate-range attraction of the baryon-baryon interaction. This mechanism is also preferred by the similarity between nuclear force and molecular force. Besides, from the phenomenological point of view, the $\Omega \Omega$ system can be best searched by the measurement of pair-momentum correlation $C(Q)$ with $Q$ being the relative momentum between two baryons produced in relativistic heavy-ion collisions [40]. Experimental confirmation of the $N \Omega$ and $\Omega \Omega$ dibaryons will provide other samples of sixquark systems than the nonstrange dibaryon $d^{*}$ [41-43]. We hope there will be more experimental collaborations involved in the search of such strange dibaryons.

\section{ACKNOWLEDGMENTS}

This work is supported partly by the National Natural Science Foundation of China under Contracts No. 11675080 , No. 11775118, and No. 11535005.
[1] A. Chodos et al., Phys. Rev. D 9, 3471 (1974).

[2] A. W. Thomas, in Advances in Nuclear Physics, edited by J. Negele (Springer, New York, 1984), pp. 1-137; T. DeGrand et al., Phys. Rev. D 12, 2060 (1975).

[3] R. Friedberg and T. D. Lee, Phys. Rev. D 15, 1694 (1977); 16, 1096 (1977); 18, 2623 (1978).

[4] T. H. R. Skyrme, Nucl. Phys. 31, 556 (1962); E. Witten, Nucl. Phys. B 160, 57 (1979); G. S. Adkins, C. R. Nappi, and E. Witten, ibid. 228, 552 (1983).

[5] A. De Rujula, H. Georgi, and S. L. Glashow, Phys. Rev. D 12, 147 (1975).

[6] N. Isgur and G. Karl, Phys. Rev. D 18, 4187 (1978); 19, 2653 (1979); 20, 1191 (1979).
[7] A. Faessler and F. Fernandez, Phys. Lett. 124, 145 (1983).

[8] I. T. Obukhovsky and A. M. Kusainov, Phys. Lett. B 238, 142 (1990).

[9] F. Fernandez, A. Valcarce, U. Straub, and Z. Faessler, J. Phys. G 19, 2013 (1993).

[10] Y. Fujiwara, C. Nakamoto, and Y. Suzuki, Phys. Rev. Lett. 76, 2242 (1996); Y. Fujiwara, T. Fujita, M. Kohno, C. Nakamoto, and Y. Suzuki, Phys. Rev. C 65, 014002 (2001).

[11] Y. W. Yu, Z. Y. Zhang, P. N. Shen, and L. R. Dai, Phys. Rev. C 52, 3393 (1995).

[12] A. Valcarce, H. Garcilazo, F. Fernández, and P. Gonzalez, Rep. Prog. Phys. 68, 965 (2005) and references therein. 
[13] M. Ablikim et al. (BES Collaboration), Phys. Lett. B 598, 149 (2004).

[14] N. Kaiser, S. Grestendorfer, and W. Weise, Nucl. Phys. A 637, 395 (1998); E. Oset, H. Toki, M. Mizobe, and T. T. Takahashi, Prog. Theo. Phys. 103, 351 (2000); M. M. Kaskulov and H. Clement, Phys. Rev. C 70, 014002 (2004).

[15] F. Wang, G. H. Wu, L. J. Teng, and T. Goldman, Phys. Rev. Lett. 69, 2901 (1992); G. H. Wu, L. J. Teng, J. L. Ping, F. Wang, and T. Goldman, Phys. Rev. C 53, 1161 (1996).

[16] P. A. M. Guichon, J. R. Stone, and A. W. Thomas, Prog. Part. Nucl. Phys. 100, 262 (2018).

[17] J. L. Ping, F. Wang, and T. Goldman, Nucl. Phys. A 657, 95 (1999); G. H. Wu et al., ibid. 673, 279 (2000); H. R. Pang, J. L. Ping, F. Wang, and T. Goldman, Phys. Rev. C 65, 014003 (2001).

[18] L. Z. Chen, H. R. Pang, H. X. Huang, J. L. Ping, and F. Wang, Phys. Rev. C 76, 014001 (2007).

[19] J. L. Ping, H. X. Huang, H. R. Pang, F. Wang, and C. W. Wong, Phys. Rev. C 79, 024001 (2009).

[20] H. Huang, J. Ping, and F. Wang, Phys. Rev. C 89, 034001 (2014).

[21] F. Wang, J. L. Ping, G. H. Wu, L. J. Teng, and T. Goldman, Phys. Rev. C 51, 3411 (1995).

[22] H. R. Pang, J. L. Ping, F. Wang, T. Goldman, and E. G. Zhao, Phys. Rev. C 69, 065207 (2004).

[23] M. Chen, H. X. Huang, J. L. Ping, and F. Wang, Phys. Rev. C 83, 015202 (2011).

[24] H. X. Huang, J. L. Ping, and F. Wang, Phys. Rev. C 92, 065202 (2015).

[25] H. X. Huang, P. Xu, J. L. Ping, and F. Wang, Phys. Rev. C 84, 064001 (2011).

[26] V. B. Kopeliovich, Nucl. Phys. A 639, 75c (1998).
[27] Z. Y. Zhang, Y. W. Yu, C. R. Ching, T. H. Ho, and Z. D. Lu, Phys. Rev. C 61, 065204 (2000).

[28] H. R. Pang, J. L. Ping, F. Wang, and T. Goldman, Phys. Rev. C 66, 025201 (2002).

[29] S. Gongyo et al. (HAL QCD Collaboration), Phys. Rev. Lett. 120, 212001 (2018).

[30] K. Morita, S. Gongyo, T. Hatsuda, T. Hyodo, Y. Kamiya, and A. Ohnishi, Phys. Rev. C 101, 015201 (2020).

[31] J. Vijande, F. Fernandez, and A. Valcarce, J. Phys. G 31, 481 (2005).

[32] H. Garcilazo, T. Fernández-Caramés, and A. Valcarce, Phys. Rev. C 75, 034002 (2007).

[33] Q. B. Li, P. N. Shen, Z. Y. Zhang, and Y. W. Yu, Nucl. Phys. A 683, 487 (2001).

[34] J. L. Ping, H. R. Pang, F. Wang, and T. Goldman, Phys. Rev. C 65, 044003 (2002).

[35] T. Goldman and R. R. Silbar, Phys. Rev. C 77, 065203 (2008).

[36] M. Kamimura, Supp. Prog. Theo. Phys. 62, 236 (1977).

[37] K. Morita, A. Ohnishi, F. Etminan, and T. Hatsuda, Phys. Rev. C 94, 031901(R) (2016).

[38] V. A. Babenko and N. M. Petrov, Phys. At. Nucl. 66, 1319 (2003)

[39] J. Adam et al. (STAR Collaboration), Phys. Lett. B 790, 490 (2019).

[40] S. Cho et al. (ExHIC Collaboration), Prog. Part. Nucl. Phys. 95, 279 (2017).

[41] M. Bashkanov et al. (CELSIUS-WASA Collaboration), Phys. Rev. Lett. 102, 052301 (2009).

[42] P. Adlarson et al. (WASA-at-COSY Collaboration), Phys. Rev. Lett. 112, 202301 (2014).

[43] M. Bashkanov et al. (A2-at-MAMI Collaboration), arXiv:1911.08309v1. 